\title{
ARTIGOS
}

\section{COMPARATIVO DE IMPACTO AMBIENTAL DE SISTEMAS CONSTRUTIVOS DE SUPERSTRUTURAS DE PONTES}

\section{COMPARATIVE OF THE ENVIRONMENTAL IMPACT OF BUILDING SYSTEMS OF BRIDGE SUPERSTRUCTURES}

\author{
TAYLANA PICCININI SCOLARO | UFSC \\ LUIZ EDUARDO PEREIRA | UTFPR \\ JAIRO TROMBETTA, M.SC. | UTFPR
}

\begin{abstract}
RESUMO
O presente estudo teve por objetivo desenvolver uma análise comparativa entre projetos de superestruturas de pontes de concreto pré-fabricado, madeira e mista aço/concreto, para três comprimentos de vão. Os projetos foram analisados quanto à geração de impacto ambiental através do desempenho apresentado em parâmetros estabelecidos dentro das categorias: consumo de energia, emissões atmosféricas e esgotamento de recursos. Foi realizada a análise do ciclo de vida dos materiais do berço ao portão. Os resultados permitiram identificar a superestrutura com o comportamento mais satisfatório em cada parâmetro. A ponte em madeira apresentou os melhores resultados em todos os parâmetros das categorias emissões atmosféricas e esgotamento de recursos naturais, por outro lado, exibiu grande consumo de energia. Em relação às demais superestruturas, o desempenho mais satisfatório em cada parâmetro das categorias variou com o comprimento do vão. Este estudo constitui uma importante ferramenta de auxílio à tomada de decisão com vistas à construção sustentável.
\end{abstract}

PALAVRAS CHAVE: Impacto ambiental; Pontes; Comparativo

\begin{abstract}
This paper aimed to develop a comparative analysis between bridge superstructures constructed of prefabricated concrete, wood and mixed steel/concrete, for three different spans length. The projects were analyzed for the generation of environmental impact through performance presented in parameters established within the categories: energy consumption, atmospheric emissions and resource depletion. The life cycle analysis of the materials from cradle to gate was carried out. The results allowed to identify the superstructure with the most satisfactory behavior in each parameter. The bridge constructed of wood showed the best results in all of the parameters in the categories atmospheric emissions and resource depletion, on the other hand, presented high energy consumption. In relation to the other superstructures, the best performance in each parameter varies according to the span length. This study is an important tool to aid decision making with a view to sustainable construction.
\end{abstract}

KEY WORDS: Environmental impact; Bridges; Comparative 


\section{INTRODUÇÃO}

Atualmente, as pontes são projetadas levando-se em consideração sobretudo aspectos de ordem econômica, técnica e de segurança. Raramente aspectos relativos ao desempenho ambiental são considerados no processo de tomada de decisão de planejamento destas estruturas. No entanto, a construção de pontes é responsável por significativo uso de energia e de matérias-primas (DU et al., 2014).

A NBR ISO 14040 (ABNT, 2009) aborda a Avaliação do Ciclo de Vida (ACV) como uma técnica de gestão ambiental em desenvolvimento que estuda os impactos ambientais ao longo da vida de um produto. Através da análise das entradas e saídas pertinentes à um sistema produtivo e da avaliação dos impactos ambientais relacionados, a ACV permite interpretar os resultados das análises e buscar medidas de melhoria em determinadas fases do ciclo de vida do produto.

Tendo em vista que as pontes, ao longo do seu ciclo de vida, podem gerar impactos ambientais, questionou-se sobre os diferentes tipos de materiais constituintes da superestrutura de uma ponte e seu desempenho frente à geração de impactos ambientais. Entende-se por superestrutura a estrutura da ponte com função de receber e transmitir as cargas que sobre ela trafegam.

Essa pesquisa teve como objetivo analisar comparativamente o desempenho ambiental de pontes executadas com diferentes sistemas construtivos - concreto pré-fabricado, madeira e mista aço/concreto - para três comprimentos de vão, através da análise do ciclo de vida dos materiais construtivos do berço ao portão.

\section{REVISÃO BIBLIOGRÁFICA}

Gervásio (2008) apresenta uma análise comparativa em termos de impactos ambientais entre duas soluções estruturais para uma ponte, uma em concreto pré-fabricado e outra mista aço/concreto. Para a solução mista, foram admitidos três diferentes cenários acerca da origem do aço estrutural: aço alto forno, aço arco elétrico e misto $(50 \%$ alto forno e $50 \%$ arco elétrico). 0 desempenho ambiental foi avaliado com base na análise de ciclo de vida, baseada nas normas ISO 14040 (2006) e ISO 14044 (2006), e com auxílio do programa de análise BEES de Lippiatt (2002). Os dados referentes aos materiais construtivos foram obtidos da base de dados do programa BEES, com exceção dos dados relativos ao aço estrutural, que foram obtidos do International Iron and Steel Institute (IISI, 2002). Foram considerados os índices: consumo de água, smog, ozônio, saúde, alteração do habitat, aquecimento global, combustíveis fósseis, eutrofização, toxicidade, poluentes do ar e acidificação. Foi analisada a fase 'cradle to gate'. Com relação aos resultados, em comparação à solução em concreto, a alternativa com aço alto forno aumentou a geração de impactos ambientais em $23 \%$, a solução em aço arco elétrico apresentou uma redução de impacto ambiental de $31 \%$ enquanto que a mista $(50 \%$ de cada processo de produção) reduziu em $4 \%$ a geração de impactos ambientais.

Castro (2012) desenvolveu uma comparação entre duas soluções construtivas para a estrutura de um edifício de $500 \mathrm{~m}^{2}$, uma de concreto armado e outra metálica. Para tal, foram consultadas sete bases de dados de Inventário de Ciclo de Vida (LCl) que fornecem valores médios de fluxos de entrada e saída associados à utilização de materiais construtivos. As categorias e parâmetros ambientais considerados pela autora foram os mesmos utilizados por este trabalho (descritos no item 3). A etapa do ciclo de vida considerada foi a 'cradle to gate'. Entretanto, após o cálculo da pontuação individual em cada um dos parâmetros, os valores foram normalizados, de maneira a possibilitar a comparação de valores em mesma unidade. Em seguida, foi determinada a pontuação de cada categoria através da atribuição de pesos a cada uma delas. Por fim, a estrutura metálica apresentou o melhor desempenho ambiental.

Ainda, uma análise comparativa do desempenho frente aos impactos ambientais de projetos equivalentes de um armazém industrial, um constituído por concreto armado e outro por estrutura metálica, foi desenvolvida por Andrade (2013), de acordo com as normas NP EN ISO 14040 e EN 15978. Os valores dos parâmetros foram obtidos da base de dados Ecoinvent, para a solução de concreto armado e de uma Declaração Ambiental de Produto (DAP) para a solução metálica. Os parâmetros ambientais considerados foram os mesmos utilizados por este trabalho e foram avaliados separadamente, atribuindo-se o mesmo peso para cada. A avalição compreendeu todo o ciclo de vida, através da elaboração de três cenários distintos: 'cradle to gate', 'cradle to grave' e 'cradle to cradle'. As duas estruturas tiveram comportamentos equivalentes nas etapas 'cradle to gate' e 'cradle to grave'. A etapa 'cradle to cradle' não foi conclusiva devido à complexidade da reciclagem ou reaproveitamento do concreto armado quando comparado ao aço.

Nesse contexto, verificou-se a ausência de estudos relativos ao uso da madeira como solução estrutural, material este que ainda é comumente encontrado em pontes de estradas vicinais da região do sudoeste do Paraná. Ademais, foi observada uma variação de resultados, bem como foi verificado que não há uma padronização metodológica para a avaliação do ciclo de vida de soluções 
construtivas. Assim, alguns estudos atribuem mesma importância aos parâmetros considerados, enquanto outros consideram pesos relativos à cada um.

Além disso, não há uma uniformidade do conjunto de parâmetros considerados na análise, dificultando a comparação entre diferentes estudos. Por fim, ressalta-se a necessidade do desenvolvimento de estudos relativos à análise de ciclo de vida de estruturas, de forma a padronizar as metodologias de avaliação, tanto na seleção, quanto na ponderação relativa dos parâmetros.

\section{METODOLOGIA}

O presente trabalho desenvolveu-se, basicamente, em três etapas. Na primeira etapa, foram selecionados os projetos das pontes já dimensionadas, constituídas uma por concreto pré-fabricado e outra por madeira, bem como elaborado um pré-dimensionamento de uma estrutura mista constituída por aço e concreto. Em seguida, determinou-se o modelo geométrico ideal para as pontes. Por fim, realizou-se o quantitativo de materiais de cada modelo para a análise ambiental.

Os projetos das pontes foram selecionados de modo a padronizar as variáveis de estudo: largura e comprimento do tabuleiro. O Manual de Construção de Pontes Municipais (contendo as especificações técnicas, projetos tipo e montagem das estruturas) foi disponibilizado pelo site do DER/PR para a ponte pré-fabricada de concreto. Para o estudo da ponte de madeira, utilizou-se do modelo descrito e dimensionado por Da Silva e Philippsen (2015). Para a ponte mista aço/concreto, por não ter sido encontrado um modelo já dimensionado com as mesmas dimensões dos demais projetos adotados, realizou-se um pré-dimensionamento com base nos resultados encontrados por Machado (2016) e Eller (2011) para perfis I em aço laminado e com auxílio do software AltoQI Eberick para o dimensionamento do tabuleiro em concreto armado. $O$ cálculo das cargas móveis foi realizado em conformidade com a NBR 7188 (ABNT, 2013). Foi adotado o trem tipo TB450 , assim como nos projetos da ponte pré-fabricada de concreto e da ponte em madeira, de forma a padronizar os projetos e prever a passagem de veículos pesados.

O trabalho teve por finalidade o estudo de pequenas pontes, usuais em estradas vicinais do sudoeste do Paraná, também denominadas pontilhões. De acordo com Machado (2016), pontilhões são pontes de pequenos vãos e larguras, com apenas uma faixa de tráfego. Com base no trabalho de Da Silva e Philippsen (2015), foi adotada a largura da ponte como sendo 6 metros. O intervalo de comprimento do vão a ser vencido foi definido com base em dados referentes às pontes existentes no município de Pato Branco (PR), obtidos no estudo de Milani (2010). Por fim, foram adotados para este estudo os vãos de 5, 7 e 9 metros, julgados comuns na região. Dessa forma, o trabaIho envolveu um total de 9 estudos, em que cada sistema construtivo (concreto pré-fabricado, madeira e misto aço/ concreto) foi analisado para 3 vãos distintos.

De posse dos projetos das pontes, realizou-se a modelagem das superestruturas utilizando o software Autodesk Revit. A partir das dimensões definidas pelos projetos e da volumetria dos elementos da ponte, foram elaboradas planilhas de cálculo pelos autores, com o auxílio do Microsoft Office Excel, de modo a obter o quantitativo de materiais de cada modelo de ponte.

Para a análise ambiental, as unidades de medida do quantitativo foram transformadas em unidade de massa (kg), para possibilitar a multiplicação das quantidades de material pelos índices de impacto ambiental. Tomaram-se como unidades funcionais as quantidades necessárias de material para compor a superestrutura das pontes: $30 \mathrm{~m}^{2}$ (6 m x $5 \mathrm{~m}), 42 \mathrm{~m}^{2}$ (6 m x $\left.7 \mathrm{~m}\right)$ e $54 \mathrm{~m}^{2}$ (6 m x $\left.9 \mathrm{~m}\right)$.

Para a análise dos impactos ambientais, tendo como diretrizes os estudos de Castro (2012) e Andrade (2013), foi considerada a fase 'cradle to gate' (do berço ao portão) como base de estudo. Assim, foram contabilizados os impactos envolvidos desde a extração da matéria-prima até a fabricação do produto, ou seja, essa etapa do ciclo de vida termina na porta da fábrica. Dessa forma, este trabaIho não contempla a análise de impactos ambientais nas fases de uso e manutenção das pontes, nem no processo de fim de vida.

Os projetos foram analisados quanto à geração de impacto ambiental através do desempenho apresentado em parâmetros estabelecidos dentro de três categorias: consumo de energia, emissões atmosféricas e esgotamento de recursos. Com base na revisão de literatura, foram selecionados os seguintes parâmetros ambientais para análise:

- Energia renovável incorporada (ER): trata sobre a preocupação em utilizar fontes renováveis;

• Energia não renovável incorporada (ENR): indica a dependência da energia não renovável incorporada de um produto, ou seja, quanto colabora para o esgotamento dos recursos;

- Potencial de aquecimento global (GWP): medida relativa da contribuição de um determinado gás para o aquecimento global em relação à mesma quantidade de $\mathrm{CO} 2$;

- Potencial de destruição de ozônio (ODP): potencial de destruição relativo de uma substância em relação à uma massa similar de CFC-11, que possui ODP igual a 1; 
- Potencial de acidificação (AP): é medido utilizando a capacidade de uma substância libertar íons $\mathrm{H}+$, causa da acidificação, ou pode ser medido em relação a uma libertação equivalente de SO2;

- Potencial de eutrofização (EP): a ocorrência de eutrofização é medida utilizando a unidade de referência de kg de nitrogênio ou equivalentes de fosfato;

- Potencial de formação de ozônio troposférico (POCP): medida da capacidade relativa de uma substância produzir ozônio na presença de NOx e luz solar. É expresso utilizando a unidade de referência kg de compostos orgânicos voláteis não metanados (NMVOC) ou kg de equivalente de etileno;
- Potencial de esgotamento de recursos abióticos (ADP): é determinado com base nas reservas remanescentes e taxa de extração. O ADP é comparado ao caso de referência antimônio (Sb).

Os valores de impactos ambientais por categoria e parâmetros considerados neste estudo foram obtidos a partir da base de dados apresentada no trabalho de Andrade (2013), conforme a Tabela 1. Estes valores têm como base as entradas e saídas de materiais, substâncias e energia associadas ao ciclo de vida dos produtos e processos.

IMPACTOS AMBIENTAIS

\begin{tabular}{|c|c|c|c|c|c|c|c|c|c|}
\hline \multirow{2}{*}{\begin{tabular}{|l} 
CATEGORIA \\
PARÂMETRO
\end{tabular}} & \multicolumn{2}{|c|}{ CONSUMO DE ENERGIA } & \multicolumn{5}{|c|}{ EMISSÕES ATMOSFÉRICA (AQUECIMENTO GLOBAL) } & \multicolumn{2}{|c|}{ ESGOTAMENTODERECURSOS } \\
\hline & 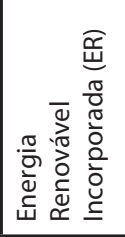 & 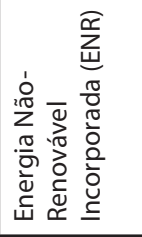 & 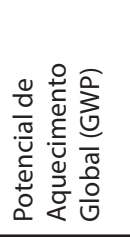 & 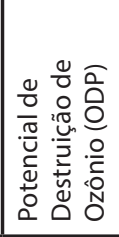 & 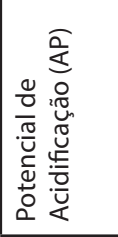 & 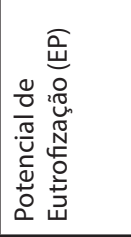 & 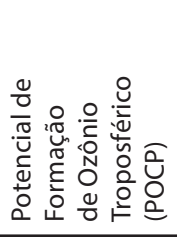 & 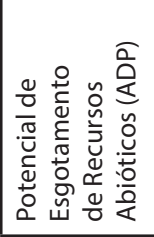 & 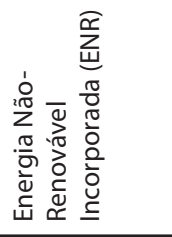 \\
\hline UNIDADE & MJ equiv & MJ equiv & $\begin{array}{l}\mathrm{Kg} \mathrm{CO} 2 \\
\text { equiv }\end{array}$ & $\begin{array}{l}\mathrm{Kg} \text { CFC } \\
11 \text { equiv }\end{array}$ & $\begin{array}{l}\mathrm{Kg} \mathrm{SO} 2 \\
\text { equiv }\end{array}$ & $\begin{array}{l}\mathrm{Kg}(\mathrm{PO} 3) 4 \\
\text { equiv }\end{array}$ & $\begin{array}{l}\text { Kg Etano } \\
\text { equiv }\end{array}$ & Kg Sb equiv & MJ equiv \\
\hline Aço vergalhão $(\mathrm{kg})$ & $1,52 \mathrm{E}-01$ & $3,27 \mathrm{E}+01$ & $1,25 \mathrm{E}+00$ & 6,16E-08 & 5,57E-03 & $1,30 \mathrm{E}-03$ & $8,29 \mathrm{E}-04$ & $1,29 \mathrm{E}-02$ & $3,27 \mathrm{E}+01$ \\
\hline Aço em perfil $(\mathrm{kg})$ & 1,16E-01 & $8,66 \mathrm{E}+00$ & $5,71 \mathrm{E}-01$ & $5,40 \mathrm{E}-08$ & $3,04 \mathrm{E}-03$ & $4,86 \mathrm{E}-04$ & $1,85 \mathrm{E}-04$ & 4,54E-03 & $8,66 \mathrm{E}+00$ \\
\hline Concreto $(\mathrm{kg})$ & $6,24 \mathrm{E}-03$ & $5,56 \mathrm{E}-01$ & 1,10E-01 & 3,55E-09 & $1,79 \mathrm{E}-04$ & $2,84 \mathrm{E}-05$ & $6,49 \mathrm{E}-06$ & $2,38 \mathrm{E}-04$ & $5,56 \mathrm{E}-01$ \\
\hline Madeira $(\mathrm{kg})$ & $1,58 \mathrm{E}+01$ & $1,98 \mathrm{E}+00$ & $-1,20 E+00$ & $1,28 \mathrm{E}-08$ & $8,05 \mathrm{E}-04$ & 1,29E-04 & $7,29 E-05$ & $1,02 \mathrm{E}-03$ & $1,98 \mathrm{E}+00$ \\
\hline
\end{tabular}

Tabela 01 - Categorias e parâmetros de cada impacto ambiental Fonte: Adaptado de Andrade (2013)

Este trabalho adotou como valores dos parâmetros para o aço dos pregos, os mesmos que os descritos por Andrade (2013) para aço vergalhão. Da mesma forma que considerou-se para madeira roliça os mesmos valores que os fornecidos pelo referido autor para madeira serrada.

\section{COMPARATIVO DE PROJETOS DE SUPE- RESTRUTURAS DE PONTES}

\subsection{Modelos de pontes}

Como este trabalho refere-se a pontes com vãos determinados de 5, 7 e $9 \mathrm{~m}$, foram estudados projetos compostos por vigas pré-fabricadas modelo DER/PR tipo "T": "TA6,50", “TA-7,50' e 'TA-9,50" (vãos livres máximos respectivamente iguais a 6, 7 e $9 \mathrm{~m}$ ), com placas e guarda-rodas em concreto armado, conforme a Figura 1 (a). Além dos elementos pré-fabricados de concreto e das armaduras de amarração, o projeto da ponte inclui concreto lançado entre vigas e concreto de cobertura, finalizando a estrutura da ponte, como exposto na Figura 1 (b). As dimensões e as especificações técnicas dos elementos construtivos citados encontram-se detalhadas no Manual de Construção de Pontes Municipais do DER. O Anexo 1 apresenta a seção transversal detalhada do tabuleiro dessa ponte.

A Figura 2 ilustra o modelo da ponte em madeira, composta por vigas roliças nas longarinas e nos guarda-rodas e por madeira serrada no tabuleiro e no rodeiro. As peças dimensionadas foram consideradas como sendo de madeira de floresta plantada da espécie 'Eucalyptus dunnii' e fixadas por pregos de aço. As dimensões das peças de madeira, conforme o tamanho do vão ponte, são especificadas no estudo de Da Silva e Philippsen (2015). O Anexo 2 apresenta a seção transversal do tabuleiro da ponte de madeira.

$\mathrm{O}$ projeto da ponte mista aço/concreto considera um tabuleiro de placas pré-fabricadas de concreto com espessura de $35 \mathrm{~cm}$, guarda-rodas pré-fabricados de concreto, duas vigas (perfis I laminados) em aço que funcionam como longarinas sustentando o tabuleiro e conectores de cisalhamento do tipo Stud Bolt, conforme mostra a Figura 3. Estes últimos fazem a ligação do tabuleiro com os perfis I. Os 
perfis I adotados foram o W 460x68 para o vão de $5 \mathrm{~m}$, o W 460x106 para o vão de $7 \mathrm{~m}$ e o W 610x140 para o vão de $9 \mathrm{~m}$. O modelo da ponte mista é apresentado na Figura 3 e a seção transversal do tabuleiro é apresentada no Apêndice 1.
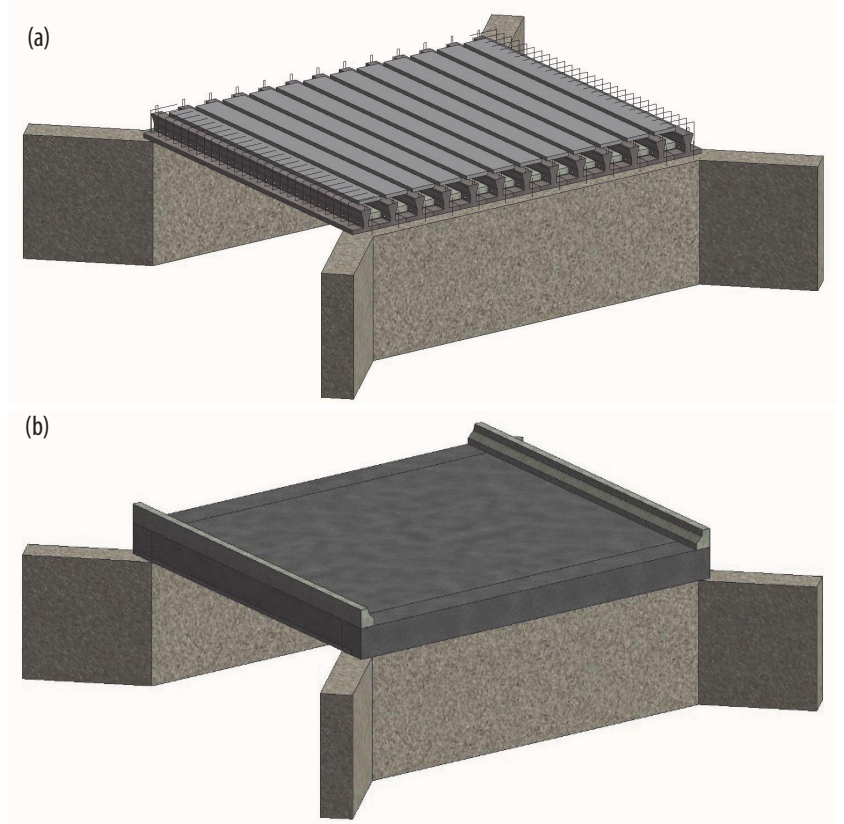

Figura 01 - (a) Elementos pré-fabricados e (b) concreto de cobertura da ponte de concreto pré-fabricado

Fonte: Autores

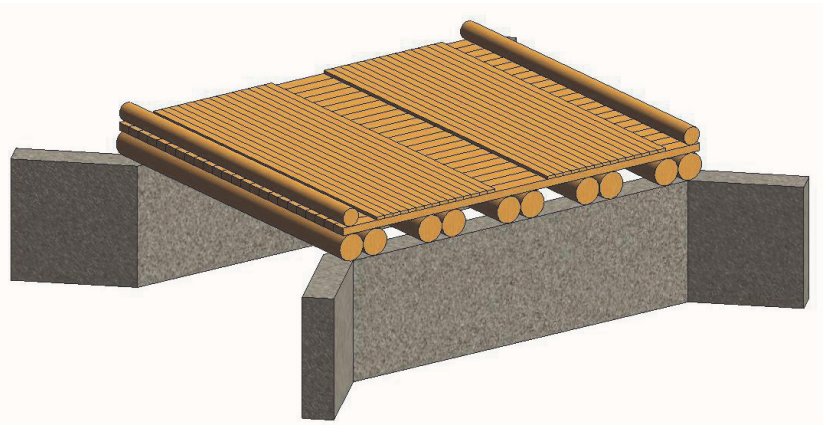

Figura 02 - Ponte em madeira

Fonte: Autores

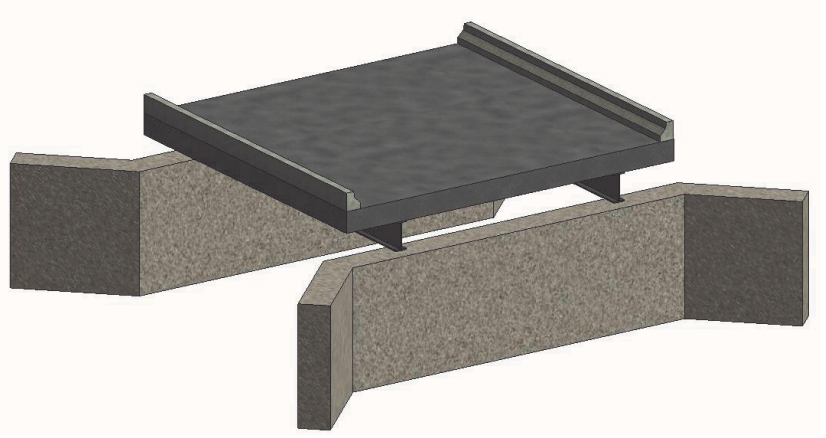

Figura 03 - Ponte mista aço/concreto Fonte: Autores

\subsection{Quantitativo de material}

A Tabela 2 apresenta o quantitativo de cada material, em kg, para cada modelo de ponte e comprimento de vão. A massa específica adotada para o concreto armado seguiu o recomendado pela NBR 6118 (ABNT, 2014).

Comparativamente, o peso é significativamente menor para o modelo de ponte em madeira, representando menos de $40 \%$ do peso da ponte em concreto pré-fabricado em todos os vãos, e correspondendo a menos de $50 \%$ ao peso da ponte mista aço/concreto em todos os comprimentos de vão. Taylor et al. (1995) também identificaram nas pontes em madeira diferentes vantagens, como o baixo peso, a facilidade de fabricação e de instalação, com a possibilidade de ser pré-fabricada.

\begin{tabular}{|l|l|l|l|}
\hline Tipo/ Dimensão & Vão 5 m & Vão 7 m & Vão 9 m \\
\hline CONCRETO PRÉ-FABRICADO \\
\hline Concreto (kg) & $41.900,00$ & $54.902,00$ & $67.904,00$ \\
\hline Aço em vergalhão (kg) & $1.699,89$ & $2.443,65$ & $3.291,41$ \\
\hline Total (kg) & $43.599,89$ & $57.345,65$ & $71.195,41$ \\
\hline MADEIRA & $11.661,00$ & $17.381,10$ & $26.854,80$ \\
\hline $\begin{array}{l}\text { Madeira Eucalyptus } \\
\text { Dunnii (kg) }\end{array}$ & 20,00 & 23,36 & 29,46 \\
\hline Prego em aço (kg) & $11.681,00$ & $17.404,46$ & $26.884,26$ \\
\hline Total (kg) & $30.645,00$ & $41.735,00$ & $52.825,00$ \\
\hline MISTA AÇO/CONCRETO \\
\hline Concreto (kg) & $1.597,20$ & $2.175,57$ & $2.753,13$ \\
\hline Aço em vergalhão (kg) & 759,48 & $1.615,72$ & $2.692,15$ \\
\hline Perfil I em aço (kg) & $33.001,68$ & $45.526,29$ & $58.270,28$ \\
\hline Total (kg)
\end{tabular}

Tabela 02 - Quantitativo de materiais por modelo de ponte e comprimento de vão Fonte: Autores

\subsection{Desempenho ambiental}

A partir da Tabela 3 é possível realizar a comparação direta do impacto ambiental gerado pelos modelos de ponte em cada parâmetro estabelecido e para cada um dos comprimentos estudados. Esta metodologia não permite que sejam estabelecidas relações entre as três categorias - que agrupam certos parâmetros - uma vez que os parâmetros são dados em diferentes unidades de medida e a comparação de diferentes unidades não é viável.

Em um estudo mais aprofundado, poderia ser realizada uma normalização de valores, de forma a converter todos os parâmetros para uma mesma unidade. Ainda, poderiam ser atribuídos diferentes pesos para cada parâmetro dentro de uma categoria, de acordo com a relevância subjetiva que possam ter dentro de determinada análise (CASTRO, 2012). 
A partir da Tabela 3, pode-se perceber que em todos os parâmetros das três categorias analisadas, com exceção do parâmetro energia renovável incorporada (ER), a ponte em madeira apresenta o melhor desempenho. Como se pode observar, a solução que apresenta melhor desempenho é aquela que exibe o menor valor em cada um dos parâmetros, indicando que a estrutura gera menor impacto ambiental no parâmetro considerado durante a etapa do ciclo de vida considerada (do berço ao portão).

Com relação à categoria de consumo de energia, em que os parâmetros ER e ENR são expressos em mesma unidade e podem, dessa forma, ser agrupados, a ponte mista aço/concreto apresenta o desempenho mais satisfatório (menor consumo de energia), seguida da ponte em concreto pré-fabricado e da ponte em madeira. A ponte em concreto pré-fabricado apresenta o menor consumo de energia renovável incorporada (ER) seguida da ponte mista aço/concreto e da ponte em madeira em todos os vãos analisados. Já no parâmetro ENR, a ponte em madeira apresenta o menor consumo, seguida da ponte mista aço/concreto e da ponte em concreto pré-fabricado. Embora o maior consumo de energia se mostre uma desvantagem ambiental para o caso da ponte em madeira, o maior consumo de fontes renováveis em detrimento das não renováveis deve ser considerado e analisado. Essa consideração deve-se ao fato de que fontes de energia renováveis podem ser aproveitadas ao longo do tempo sem possibilidade de esgotamento, enquanto que a utilização de fontes de energia não renováveis depende da limitação de recursos existentes.
Na categoria emissões atmosféricas, a ponte em madeira apresenta melhores resultados em todos os parâmetros. Nos parâmetros GWP e POCP, o melhor, mediano e pior desempenho são relativos, respectivamente, à ponte em madeira, mista aço/concreto e ponte em concreto pré-fabricado. Já para os demais parâmetros (ODP, AP e EP), o segundo melhor desempenho em cada parâmetro é atribuído à ponte mista aço/concreto no vão de $5 \mathrm{~m}$ e à ponte em concreto pré-fabricado nos vãos de 7 e $9 \mathrm{~m}$. A ponte em madeira apresenta valores negativos de impacto ambiental no parâmetro potencial de aquecimento global (GWP) devido à capacidade de aprisionamento de $\mathrm{CO} 2$ da madeira.

Da mesma forma, na categoria esgotamento de recursos a ponte em madeira apresenta desempenho mais satisfatório em todos os parâmetros. No parâmetro ENR, o segundo melhor comportamento é atribuído à ponte mista aço/concreto. Já no parâmetro ADP, o desempenho das pontes em concreto pré-fabricado e da ponte mista aço/concreto varia com o vão, sendo a ponte em concreto pré-fabricado a que apresenta menor impacto ambiental nos vãos 7 e $9 \mathrm{~m}$.

Nesse sentido, ressalta-se a importância do detalhamento dos projetos anteriormente à realização de análises comparativas, uma vez que a escolha das práticas construtivas e dos materiais de construção utilizados influenciam significativamente os resultados. Isto foi evidenciado pela variação no desempenho ambiental apresentado por um mesmo projeto de ponte, quando avaliado sob diferentes características geométricas (avaliação de diferentes comprimentos de vão e consequentemente diferentes áreas de tabuleiro).

\begin{tabular}{|l|l|l|l|l|l|l|}
\hline QUANTIFICAÇÃO DE IMPACTOS AMBIENTAIS DOS MATERIAIS DO BERÇO AO PORTÃO \\
\hline $\begin{array}{l}\text { MODELO } \\
\text { DE PONTE }\end{array}$
\end{tabular}




\begin{tabular}{|c|c|c|c|c|c|c|}
\hline \multirow{7}{*}{$\begin{array}{l}\text { Madeira } \\
\text { (continuação) }\end{array}$} & \multirow{5}{*}{$\begin{array}{l}\text { Emissões } \\
\text { Atmosféricas } \\
\text { (Aquecimento } \\
\text { Global) }\end{array}$} & Potencial de Aquecimento Global (GWP) & $\mathrm{Kg} \mathrm{CO} 2$ equiv & $-1,40 E+04$ & $-2,08 \mathrm{E}+04$ & $-3,22 \mathrm{E}+04$ \\
\hline & & Potencial de Destruição do Ozônio (ODP) & Kg CFC 11 equiv & $1,50 \mathrm{E}-04$ & 2,24E-04 & $3,46 \mathrm{E}-04$ \\
\hline & & Potencial de Acidificação (AP) & $\mathrm{Kg} \mathrm{SO} 2$ equiv & $9,50 \mathrm{E}+00$ & $1,41 \mathrm{E}+01$ & $2,18 \mathrm{E}+01$ \\
\hline & & Potencial de Eutrofização (EP) & $\mathrm{Kg}$ (PO3)4 equiv & $1,53 \mathrm{E}+00$ & $2,27 \mathrm{E}+00$ & $3,50 \mathrm{E}+00$ \\
\hline & & $\begin{array}{l}\text { Potencial de Formação de Ozônio } \\
\text { Troposférico (POCP) }\end{array}$ & Kg Etano equiv & 8,67E-01 & $1,29 E+00$ & $1,98 \mathrm{E}+00$ \\
\hline & \multirow[t]{2}{*}{$\begin{array}{l}\text { Esgotamento } \\
\text { de Recursos }\end{array}$} & $\begin{array}{l}\text { Potencial de Esgotamento de } \\
\text { Recursos Abióticos (ADP) }\end{array}$ & Kg Sb equiv & $1,22 \mathrm{E}+01$ & $1,80 \mathrm{E}+01$ & $2,78 \mathrm{E}+01$ \\
\hline & & Energia Não Renovável Incorporada (ENR) & MJ equiv & $2,37 \mathrm{E}+04$ & $3,52 E+04$ & $5,41 \mathrm{E}+04$ \\
\hline \multirow{9}{*}{$\begin{array}{l}\text { Mista aço/ } \\
\text { concreto }\end{array}$} & \multirow{2}{*}{$\begin{array}{l}\text { Consumo } \\
\text { de Energia }\end{array}$} & Energia Renovável Incorporada (ER) & MJ equiv & $5,22 \mathrm{E}+02$ & $7,79 \mathrm{E}+02$ & $1,06 \mathrm{E}+03$ \\
\hline & & Energia Não Renovável Incorporada (ENR) & MJ equiv & $7,58 \mathrm{E}+04$ & $1,08 \mathrm{E}+05$ & $1,43 \mathrm{E}+05$ \\
\hline & \multirow{5}{*}{$\begin{array}{l}\text { Emissões } \\
\text { Atmosféricas } \\
\text { (Aquecimento } \\
\text { Global) }\end{array}$} & Potencial de Aquecimento Global (GWP) & $\mathrm{Kg} \mathrm{CO} 2$ equiv & $5,80 \mathrm{E}+03$ & $8,23 E+03$ & $1,08 \mathrm{E}+04$ \\
\hline & & Potencial de Destruição do Ozônio (ODP) & Kg CFC 11 equiv & $2,48 \mathrm{E}-04$ & 3,69E-04 & $5,02 \mathrm{E}-04$ \\
\hline & & Potencial de Acidificação (AP) & $\mathrm{Kg} \mathrm{SO} 2$ equiv & $1,67 \mathrm{E}+01$ & $2,45 \mathrm{E}+01$ & $3,30 \mathrm{E}+01$ \\
\hline & & Potencial de Eutrofização (EP) & $\mathrm{Kg}$ (PO3)4 equiv & $3,32 \mathrm{E}+00$ & $4,80 \mathrm{E}+00$ & $6,39 \mathrm{E}+00$ \\
\hline & & $\begin{array}{l}\text { Potencial de Formação de Ozônio } \\
\text { Troposférico (POCP) }\end{array}$ & Kg Etano equiv & $1,66 \mathrm{E}+00$ & $2,37 \mathrm{E}+00$ & $3,12 \mathrm{E}+00$ \\
\hline & \multirow[t]{2}{*}{$\begin{array}{l}\text { Esgotamento } \\
\text { de Recursos }\end{array}$} & $\begin{array}{l}\text { Potencial de Esgotamento de } \\
\text { Recursos Abióticos (ADP) }\end{array}$ & Kg Sb equiv & $3,13 \mathrm{E}+01$ & $4,53 \mathrm{E}+01$ & $6,03 \mathrm{E}+01$ \\
\hline & & Energia Não Renovável Incorporada (ENR) & MJ equiv & $7,58 \mathrm{E}+04$ & $1,08 \mathrm{E}+05$ & $1,43 \mathrm{E}+05$ \\
\hline
\end{tabular}

Tabela 03 - Comparativo ambiental das pontes em concreto pré-fabricado, madeira e mista aço/concreto Fonte: Autores

\section{CONCLUSÕES}

Acerca do peso de todos os materiais utilizados em cada projeto de superestrutura, a ponte em madeira apresenta-se como a mais leve em todos os vãos, seguida da ponte mista aço/concreto e da ponte em concreto pré-fabricado.

Com relação à análise ambiental, pode-se dizer que a ponte em madeira exibe os melhores resultados em todos os parâmetros das categorias emissões atmosféricas e esgotamento de recursos naturais, contudo, implica em grande consumo de energia. Isto significa que contabilizando todos os processos envolvidos na extração da matéria-prima e na transformação em produtos de construção, o sistema construtivo em madeira apresenta menor impacto ambiental relacionado com as emissões atmosféricas e com o esgotamento de recursos naturais, entretanto, resulta em maior consumo de energia.

Em relação as demais pontes - que em todos os parâmetros, com exceção do parâmetro ER, apresentam resultados menos satisfatórios que a ponte em madeira - em alguns parâmetros a ponte em concreto pré-fabricado assume melhor desempenho, em outros a mista aço/concreto tem melhor desempenho, fato que varia com o vão estabelecido.

Constatou-se que a análise ambiental realizada por este estudo é uma ferramenta efetiva de comparação entre tipologias construtivas, pois permite adaptar diferentes soluções ao processo. Entretanto, ainda são necessários estudos complementares envolvendo as demais etapas do ciclo de vida não compreendidas pelos limites deste trabalho, considerando as fases de uso, manutenção, bem como processos de reciclagem e aproveitamento dos materiais ao fim do ciclo. Nesse sentido, sugere-se que componentes relacionados à durabilidade das estruturas sejam abordadas por trabalhos futuros.

Destaca-se que esta metodologia de estudo não determina qual é a melhor estrutura ou o melhor processo em termos de rentabilidade e funcionalidade, mas pode ser vista como ferramenta de apoio para a tomada de decisão acerca da construção sustentável. Ressalta-se ainda que análises comparativas devem ser realizadas dentro de cada contexto e época específica e deve-se considerar a harmonia entre os três pilares da sustentabilidade - as dimensões ambiental, econômica e social. Por fim, entende-se que a elaboração de comparativos de projetos distintos constitui um importante recurso para a tomada de decisão com vistas à sustentabilidade na construção civil.

\section{REFERÊNCIAS}

ABNT. NBR ISO 14040: Gestão ambiental - Avaliação do ciclo de vida - Princípios e estrutura. Rio de Janeiro. 2009.

NBR 6118: Projeto de estruturas de concreto - Procedimento. Rio de Janeiro. 2014.

NBR 7188: Carga móvel rodoviária e de pedestres em pontes, viadutos, passarelas e outras 
estruturas. Rio de Janeiro. 2013.

ANDRADE, T. F. R. Integração da análise ciclo de vida nas práticas de projetos de edifícios. Dissertação (Mestrado em Engenharia Civil) - Faculdade de Engenharia da Universidade do Porto, Porto, 2013. Disponível em: https://hdl.handle.net/10216/76483.

CASTRO, R. R. T. L. Análise da sustentabilidade de estruturas: aço vs betão. Tese (Doutorado em Construção e Reabilitação Sustentáveis) Universidade do Minho, Braga, 2012. Disponível em: http://hdl.handle.net/1822/24766.

DA SILVA, F. J.; PHILIPPSEN, M. Estudo e concepção de modelo geométrico e estrutural de ponte em vigas de madeira roliça de eucalipto para estradas vicinais da Região Sudoeste do Estado do Paraná. Trabalho de Conclusão de Curso (Graduação em Engenharia Civil) - Universidade Tecnológica Federal do Paraná, Pato Branco, 2015. Disponível em: http://repositorio.roca.utfpr.edu.br/jspui/handle/1/7146.

DER. Departamento de Estradas de Rodagem. Construção de Pontes Municipais. Especificações técnicas, projetos tipo e montagem. 1999.

DU, G. et al. Life cycle assessment as a decision support tool for bridge procurement: environmental impact comparison among five bridge designs. Int $J$ Life Cycle Assess, n.19, p.1948-1964, 2014. Disponível em: $\quad$ https://link.springer.com/article/10.1007/ s11367-014-0797-z.

ELLER, P. R. Pré-Dimensionamento de Vigas Mistas de Aço e Concreto para Pontes de Pequeno Porte. Dissertação (Mestrado em Ciências da Engenharia Civil) - Universidade Federal de Ouro Preto, Ouro Preto, 2011. Disponível em: https://www.propec.ufop. br/uploads/propec_2016/teses/arquivos/tese175.pdf. GERVÁSIO, H. A sustentabilidade do aço e das estruturas metálicas. In: Congresso Latino-Americano da construção metálica CONSTRUMETAL. São Paulo, Brasil. 2008. Disponível em: https://www.abcem.org. br/construmetal/2008/downloads/PDFs/27_Helena_ Gervasio.pdf.

MACHADO, L. R. Pontilhões de madeira: substituição por pontes em estruturas mistas. Trabalho de Conclusão de Curso (Graduação em Engenharia Civil) - Universidade Federal do Rio Grande do Sul, Porto Alegre, 2016. Disponível em: http://hdl.handle. net/10183/148732.

MILANI, C. J. Subsídios para o diagnóstico das pontes do sistema viário do município de Pato Branco - Paraná. 216f. Dissertação (Mestrado em Engenharia)
-Universidade de Passo Fundo, Passo Fundo, 2010. Disponível em: http://tede.upf.br:8080/jspui/handle/ tede/260.

TAYLOR, S. E.; RITTER, M.A; MURPHY, G. L. Portable glulam timber bridge design for low-volume forest roads. In: Proceedings of the 6th International conference on low-volume roads, p. 25-29, 1995. Disponível em: https://pdfs.semanticscholar.org/6dee/e0b6900db575d1861450e31814c8dbe3ddbf.pdf. 


\section{ANEXO 1}

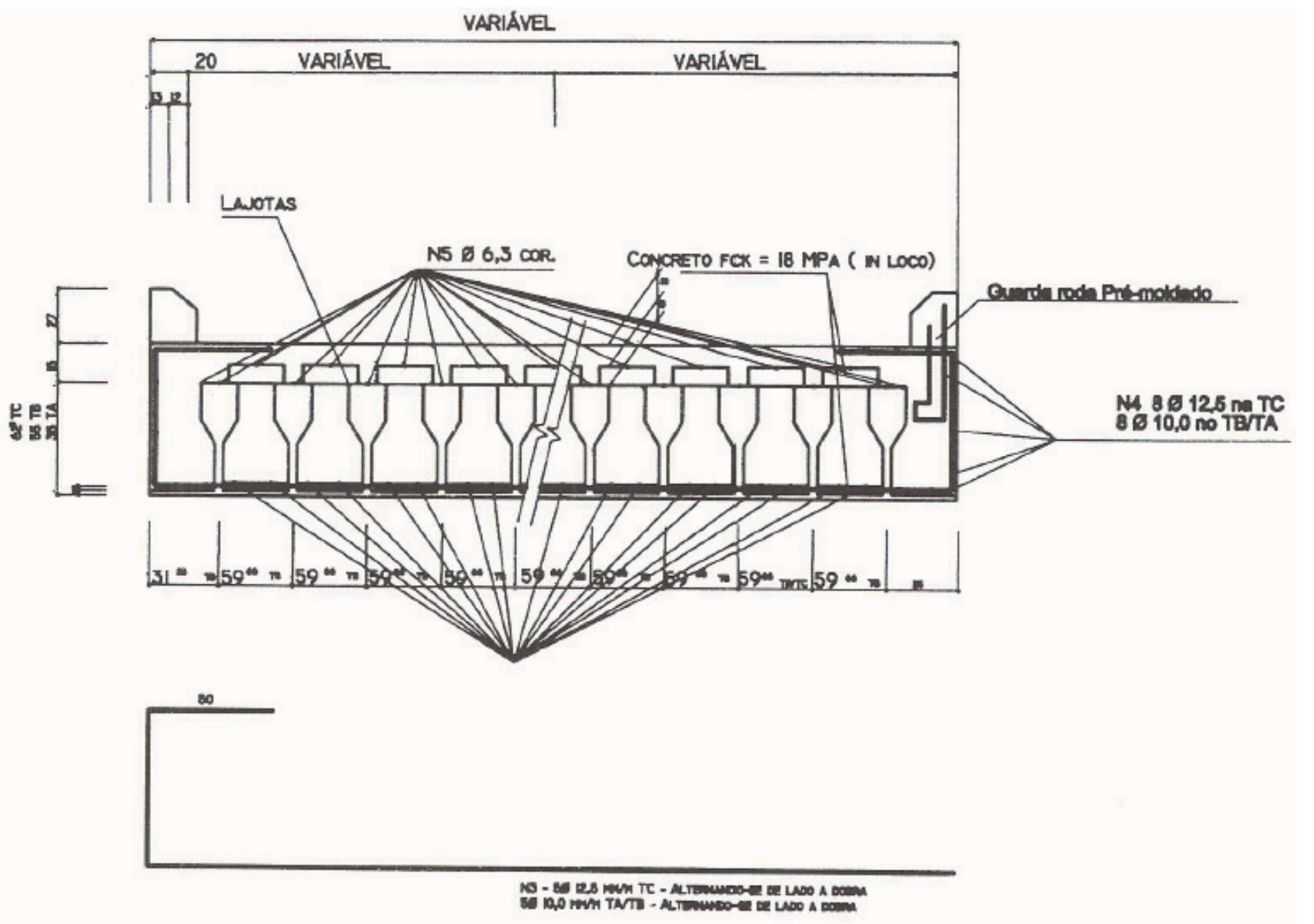

Figura 04 - Seção transversal da ponte de concreto

Fonte: Autores

\section{ANEXO 2}

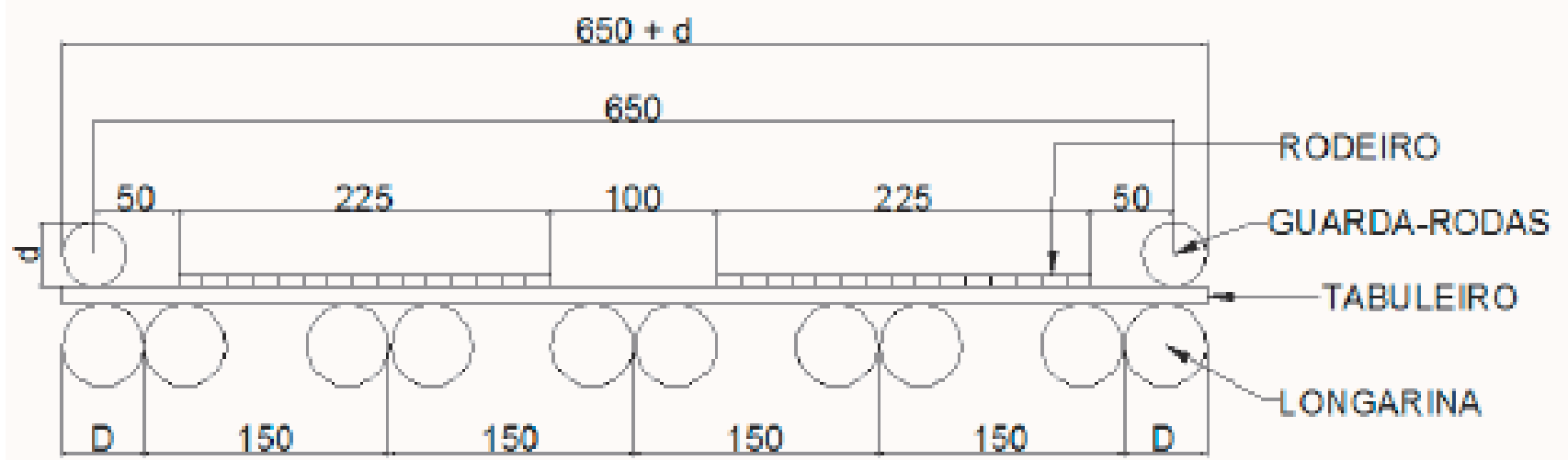

Figura 05 - Seção transversal da ponte em madeira

Fonte: Da Silva e Philippsen (2015) 


\section{AUTORES}

ORCID: https://orcid.org/0000-0003-4296-0686

TAYLANA PICCININI SCOLARO | Universidade Federal de Santa Catarina | Engenharia Civil | Florianópolis, SC - Brasil | Correspondência para: Departamento de Engenharia Civil da Universidade Federal de Santa Catarina, R. João Pio Duarte Silva, s/n - Córrego Grande, Florianópolis - SC, 88040-900 | e-maill: taylanaps@hotmail.com

ORCID: -

LUIZ EDUARDO PEREIRA | Universidade Tecnológica Federal do Paraná | Engenharia Civil | Pato Branco, PR - Brasil | Correspondência para: Departamento de Engenharia Civil da Universidade Tecnológica Federal do Paraná, Via do conhecimento, km 1 - Pato Branco - Pr, 85503-390 | e-maill: luiz_eduu@hotmail.com

ORCID: -

JAIRO TROMBETTA, M.Sc. | Universidade Tecnológica Federal do Paraná | Engenharia Civil | Pato Branco, PR - Brasil | Correspondência para: Departamento de Engenharia Civil da Universidade Tecnológica Federal do Paraná, Via do conhecimento, km 1 - Pato Branco - Pr, 85503-390| E-maill: jairotrombetta@gmail.com

\section{COMO CITAR ESTE ARTIGO}

SCOLARO, Taylana Piccinini; PEREIRA, Luiz Eduardo; TROMBETTA, Jairo. Comparativo de Impacto Ambiental de Sistemas Construtivos de Superstruturas de Pontes. MIX Sustentável, [S.I.], v. 6, n. 3, p. 107-116, jun. 2020. ISSN 24473073. Disponível em:<http://www. nexos.ufsc.br/index.php/mixsustentavel>. Acesso em: dia mês. ano. doi:https://doi.org/10.29183/2447-3073. MIX2020.v6.n3.107-116.

DATA DE ENVI0: 27/01/2020

DATA DE ACEITE: $21 / 05 / 2020$ 